\title{
Two-log-convexity of the Catalan-Larcombe-French sequence
}

Brian Y Sun ${ }^{*}$ and Baoyindureng Wu

${ }^{\text {*Correspondence: }}$

brianys1984@126.com

College of Mathematics and System Science, Xinjiang University, Urimqi, 830046, P.R. China

\begin{abstract}
The Catalan-Larcombe-French sequence $\left\{P_{n}\right\}_{n \geq 0}$ arises in a series expansion of the complete elliptic integral of the first kind. It has been proved that the sequence is log-balanced. In the paper, by exploring a criterion due to Chen and Xia for testing 2-log-convexity of a sequence satisfying three-term recurrence relation, we prove that the new sequence $\left\{P_{n}^{2}-P_{n-1} P_{n+1}\right\}_{n \geq 1}$ are strictly log-convex and hence the Catalan-Larcombe-French sequence is strictly 2-log-convex.
\end{abstract}

MSC: 05A20; 11B37; 11B83

Keywords: log-balanced sequence; log-convex sequence; log-concave sequence; the Catalan-Larcombe-French sequence; three-term recurrence

\section{Introduction}

This paper is concerned with the log-behavior of the Catalan-Larcombe-French sequence. To begin with, let us recall that a sequence $\left\{z_{n}\right\}_{n \geq 0}$ is said to be log-concave if

$$
z_{n}^{2} \geq z_{n+1} z_{n-1}, \quad \text { for } n \geq 1,
$$

and it is log-convex if

$$
z_{n}^{2} \leq z_{n+1} z_{n-1}, \quad \text { for } n \geq 1
$$

Meanwhile, the sequence $\left\{z_{n}\right\}_{n \geq 0}$ is called strictly log-concave (resp. log-convex) if the inequality in (1.1) (resp. (1.2)) is strict for all $n \geq 1$. We call $\left\{z_{n}\right\}_{n \geq 0} \log$-balanced if the sequence itself is log-convex while $\left\{\frac{z_{n}}{n !}\right\}_{n \geq 0}$ is $\log$-concave.

Given a sequence $A=\left\{z_{n}\right\}_{n \geq 0}$, define the operator $\mathcal{L}$ by

$$
\mathcal{L}(A)=\left\{s_{n}\right\}_{n \geq 0},
$$

where $s_{n}=z_{n-1} z_{n+1}-z_{n}^{2}$ for $n \geq 1$. We say that $\left\{z_{n}\right\}_{n \geq 0}$ is $k$-log-convex (resp. $k$-log-concave) if $\mathcal{L}^{j}(A)$ is log-convex (resp. $\log$-concave) for all $j=0,1, \ldots, k-1$, and that $A=\left\{z_{n}\right\}_{n \geq 0}$ is $\infty$ log-convex (resp. $\infty$-log-concave) if $\mathcal{L}^{k}(A)$ is log-convex (resp. log-concave) for any $k \geq 0$. Similarly, we can define strict $k$-log-concavity or strict $k$-log-convexity of a sequence.

(c) 2015 Sun and Wu. This article is distributed under the terms of the Creative Commons Attribution 4.0 International License (http://creativecommons.org/licenses/by/4.0/), which permits unrestricted use, distribution, and reproduction in any medium, provided you give appropriate credit to the original author(s) and the source, provide a link to the Creative Commons license, and indicate if changes were made. 
It is worthy to mention that besides that they are fertile sources of inequalities, logconvexity and log-concavity have many applications in some different mathematical disciplines, such as geometry, probability theory, combinatorics, and so on. See the surveys due to Brenti [1] and Stanley [2] for more details. Additionally, it is clear that the log-balancedness implies the log-convexity and a sequence $\left\{z_{n}\right\}_{n \geq 0}$ is log-convex (resp. $\log$-concave) if and only if its quotient sequence $\left\{\frac{z_{n}}{z_{n-1}}\right\}_{n \geq 1}$ is nondecreasing (resp. nonincreasing). It is also known that the quotient sequence of a log-balanced sequence does not grow too fast. Therefore, log-behavior are important properties of combinatorial sequences and they are instrumental in obtaining the growth rate of a sequence. Hence the log-behaviors of a sequence deserves to be investigated.

In this paper, we investigate the 2-log-behavior of the Catalan-Larcombe-French sequence, denoted by $\left\{P_{n}\right\}_{n \geq 0}$, which arises in connection with series expansions of the complete elliptic integrals of the first kind [3, 4]. To be precise, for $0<|c|<1$,

$$
\int_{0}^{\pi / 2} \frac{1}{\sqrt{1-c^{2} \sin ^{2} \theta}} d \theta=\frac{\pi}{2} \sum_{n=0}^{\infty}\left(\frac{1-\sqrt{1-c^{2}}}{16}\right)^{n} P_{n}
$$

Furthermore, the numbers $P_{n}$ can be written as the following sum:

$$
P_{n}=2^{n} \sum_{k=0}^{n}(-4)^{i}\left(\begin{array}{c}
n-k \\
k
\end{array}\right)\left(\begin{array}{c}
2 n-2 k \\
n-k
\end{array}\right)^{2}
$$

see [5], A05317. Besides, the number $P_{n}$ satisfies three-term recurrence relations [4] as follows:

$$
(n+1)^{2} P_{n+1}=8\left(3 n^{2}+3 n+1\right) P_{n}-128 n^{2} P_{n-1}, \quad \text { for } n \geq 1,
$$

with the initial values $P_{0}=1$ and $P_{1}=8$.

Recently, Zhao [4] studied the log-behavior of the Catalan-Larcombe-French sequence and proved that the sequence $\left\{P_{n}\right\}_{n \geq 0}$ is log-balanced. What is more, the CatalanLarcombe-French sequence has many interesting properties and the reader can refer [3, $4,6]$. In the sequel, we study the 2-log-behavior of the sequences and obtain the following result.

Theorem 1.1 The Catalan-Larcombe-French sequence $\left\{P_{n}\right\}_{n \geq 0}$ is strictly 2-log-convex, that is,

$$
\mathcal{P}_{n}^{2}<\mathcal{P}_{n-1} \mathcal{P}_{n+1}
$$

where $\mathcal{P}_{n}=P_{n}^{2}-P_{n-1} P_{n+1}$.

We will give our proof of Theorem 1.1 in the third section by utilizing a testing criterion, which is proposed by Chen and Xia [7].

To make this paper self-contained, let us recall their criterion. 
Theorem 1.2 (Chen and Xia [7]) Suppose $\left\{z_{n}\right\}_{n \geq 0}$ is a positive log-convex sequence that satisfies the following three-term recurrence relation:

$$
z_{n}=a(n) z_{n-1}+b(n) z_{n-2}, \quad \text { for } n \geq 2 .
$$

Let

$$
\begin{aligned}
c_{0}(n)= & -b^{2}(n+1)\left[a^{2}(n+2)+b(n+1)-a(n+2) a(n+3)-b(n+3)\right] ; \\
c_{1}(n)= & b(n+1)[2 a(n+2) b(n+1)+2 a(n+3) a(n+2) a(n+1) \\
& +a(n+3) b(n+2)+2 a(n+1) b(n+3)-2 a^{2}(n+2) a(n+1) \\
& -2 a(n+2) b(n+2)-3 a(n+1) b(n+1)] ; \\
c_{2}(n)= & 4 a(n+1) a(n+2) b(n+1)+2 b(n+1) b(n+2)+a^{2}(n+1) a(n+2) a(n+3) \\
& +a(n+1) a(n+3) b(n+2)+a^{2}(n+1) b(n+3)-3 a^{2}(n+1) b(n+1) \\
& -a(n+3) a(n+2) b(n+1)-a^{2}(n+2) a^{2}(n+1)-b(n+3) b(n+1) \\
& -2 a(n+2) a(n+1) b(n+2)-b^{2}(n+2) ; \\
c_{3}(n)= & 2 a^{2}(n+1) a(n+2)+2 a(n+1) b(n+2)-a(n+1) b(n+3)-a^{3}(n+1) \\
& -a(n+1) a(n+2) a(n+3)-a(n+3) b(n+2) ;
\end{aligned}
$$

and

$$
\Delta(n)=4 c_{2}^{2}(n)-12 c_{1}(n) c_{3}(n)
$$

Assume that $c_{3}(n)<0$ and $\Delta(n) \geq 0$ for all $n \geq N$, where $N$ is a positive integer. If there exist $f_{n}$ and $g_{n}$ such that, for all $n \geq N$,

(I) $f_{n} \leq \frac{z_{n}}{z_{n-1}} \leq g_{n}$

(II) $f_{n} \geq \frac{-2 c_{2}(n)-\sqrt{\Delta(n)}}{6 c_{3}(n)}$;

(III) $c_{3}(n) g_{n}^{3}+c_{2}(n) g_{n}^{2}+c_{1}(n) g_{n}+c_{0}(n) \geq 0$,

then we see that $\left\{z_{n}\right\}_{n \geq N}$ is 2-log-convex, that is, for $n \geq N$,

$$
\left(z_{n-1} z_{n+1}-z_{n}^{2}\right)\left(z_{n+1} z_{n+3}-z_{n+2}^{2}\right)>\left(z_{n} z_{n+2}-z_{n+1}^{2}\right)^{2}
$$

With respect to the theory in this field, it should be mentioned that the log-behavior of a sequence which satisfies a three-term recurrence has been extensively studied; see Liu and Wang [8], Chen et al. [9, 10], Liggett [11], Došlić [12], etc.

\section{Bounds for $\frac{P_{n}}{P_{n-1}}$}

Before proving Theorem 1.1, we need the following two lemmas.

Lemma 2.1 Let

$$
f_{n}=\frac{232 n}{15(n+2)} \text {, }
$$


and $P_{n}$ be the sequence defined by the recurrence relation (1.3). Then we have, for all $n \geq 1$,

$$
\frac{P_{n}}{P_{n-1}}>f_{n}
$$

Proof We proceed the proof by induction. First note that, for $n=1$ and $n=2$, we have $\frac{P_{1}}{P_{0}}=8>\frac{232}{45}$ and $\frac{P_{2}}{P_{1}}=10>\frac{464}{60}$. Assume that the inequality (2.1) is valid for $n \leq k$. We will show that

$$
\frac{P_{k+1}}{P_{k}}>f_{k+1}
$$

By the recurrence (1.3), we have

$$
\begin{aligned}
\frac{P_{k+1}}{P_{k}} & =\frac{8\left(3 k^{2}+3 k+1\right)}{(k+1)^{2}}-\frac{128 k^{2}}{(k+1)^{2}} \frac{P_{k-1}}{P_{k}}>\frac{8\left(3 k^{2}+3 k+1\right)}{(k+1)^{2}}-\frac{128 k^{2}}{(k+1)^{2}} \frac{1}{f_{k}} \\
& =\frac{8\left(57 k^{2}+27 k+29\right)}{29(k+1)^{2}} \\
& >f_{k+1},
\end{aligned}
$$

in which the last inequality follows by

$$
\begin{aligned}
\frac{8\left(57 k^{2}+27 k+29\right)}{29(k+1)^{2}}-f_{k+1} & =\frac{8\left(14 k^{3}+447 k^{2}-873 k+464\right)}{435(k+1)^{2}(k+3)} \\
& >0
\end{aligned}
$$

for all $k \geq 1$. This completes the proof.

Lemma 2.2 Let

$$
g_{n}=16-\frac{16}{n}-\frac{16}{n^{3}}
$$

and $P_{n}$ be the sequence defined by the recurrence relation (1.3). Then we have, for all $n \geq 6$,

$$
\frac{P_{n}}{P_{n-1}} \leq g_{n}
$$

Proof First note that, for $n=6$, we have $\frac{P_{6}}{P_{5}}=\frac{3,562}{269}<g_{6}=\frac{358}{27}$. Assume that, for $k \geq 6$, the inequality (2.2) is valid for $n \leq k$. We will show that

$$
\frac{P_{k+1}}{P_{k}}<g_{k+1}
$$

By the recurrence (1.3), we have

$$
\begin{aligned}
\frac{P_{k+1}}{P_{k}} & =\frac{8\left(3 k^{2}+3 k+1\right)}{(k+1)^{2}}-\frac{128 k^{2}}{(k+1)^{2}} \frac{P_{k-1}}{P_{k}}<\frac{8\left(3 k^{2}+3 k+1\right)}{(k+1)^{2}}-\frac{128 k^{2}}{(k+1)^{2}} \frac{1}{g_{k}} \\
& =\frac{8\left(2 k^{5}-2 k^{3}-4 k^{2}-3 k-1\right)}{(k+1)^{2}\left(k^{3}-k^{2}-1\right)} .
\end{aligned}
$$


Consider

$$
\frac{8\left(2 k^{5}-2 k^{3}-4 k^{2}-3 k-1\right)}{(k+1)^{2}\left(k^{3}-k^{2}-1\right)}-g_{k+1}=-\frac{8\left(5 k^{2}+2 k+3\right)}{(k+1)^{3}\left(k^{3}-k^{2}-1\right)}<0,
$$

for all $k \geq 2$. So we see that, for all $n \geq 6$, the inequality (2.2) holds by induction.

With the above lemmas in hand, we are now in a position to prove our main result in the next section.

\section{Proof of Theorem 1.1}

In this section, by using the criterion of Theorem 1.2, we can show that the CatalanLarcombe-French sequence is strictly 2-log-convex.

To begin with, the following lemma, which is obtained by Zhao [4], is indispensable for us.

Lemma 3.1 (Zhao [4]) The Catalan-Larcombe-French sequence is log-balanced.

By the definition of log-balanced sequence, we know that $\left\{P_{n}\right\}_{n \geq 0}$ is $\log$-convex.

Proof of Theorem 1.1 By Lemma 3.1, it suffices for us to show that

$$
\left(P_{n-1} P_{n+1}-P_{n}^{2}\right)\left(P_{n+1} P_{n+3}-P_{n+2}^{2}\right)-\left(P_{n} P_{n+2}-P_{n+1}^{2}\right)^{2}>0 .
$$

According to the recurrence relation (1.3), we see that

$$
\begin{aligned}
& a(n)=\frac{8\left(3 n^{2}-3 n+1\right)}{n^{2}} ; \\
& b(n)=-\frac{128(n-1)^{2}}{n^{2}} .
\end{aligned}
$$

By taking $a(n), b(n)$ in $c_{0}, \ldots, c_{3}$, we can obtain

$$
\begin{aligned}
c_{3}(n)= & -\frac{512}{(n+1)^{6}(n+2)^{2}(n+3)^{2}} \\
& \times\left(3 n^{8}+5 n^{7}-27 n^{6}-32 n^{5}+112 n^{4}+234 n^{3}+177 n^{2}+63 n+9\right) \\
< & 0,
\end{aligned}
$$

for all $n \geq 1$. Besides, we have to verify that, for some positive integer $N$, the conditions (II) and (III) in Theorem 1.2 hold for all $n \geq N$. That is,

$$
\begin{aligned}
& f_{n} \geq \frac{-2 c_{2}(n)-\sqrt{\Delta(n)}}{6 c_{3}(n)} ; \\
& c_{3}(n) g_{n}^{3}+c_{2}(n) g_{n}^{2}+c_{1}(n) g_{n}+c_{0}(n) \geq 0 .
\end{aligned}
$$

Let

$$
\delta(n)=-6 c_{3}(n) f_{n}-2 c_{2}(n)
$$


and

$$
f\left(g_{n}\right)=c_{3}(n) g_{n}^{3}+c_{2}(n) g_{n}^{2}+c_{1}(n) g_{n}+c_{0}(n)
$$

To show (3.1), it is equivalent to show that, for some positive integers $N, \delta(n) \geq 0$ and $\delta^{2}(n) \geq \Delta(n)$. By calculating, we easily find that, for all $n \geq 1$,

$$
\begin{aligned}
\delta(n)= & \frac{8,192}{5(n+1)^{6}(n+2)^{4}(n+3)^{2}}\left(32 n^{10}+129 n^{9}+472 n^{8}+3,556 n^{7}+12,157 n^{6}\right. \\
& \left.+17,632 n^{5}+10,550 n^{4}+1,293 n^{3}-1,500 n^{2}-798 n-135\right) \\
\geq & 0
\end{aligned}
$$

and for all $n \geq 3$,

$$
\begin{aligned}
\delta^{2}(n)-\Delta(n)= & \frac{6,7108,864 n}{25(n+3)^{4}(n+2)^{7}(n+1)^{12}}\left(699 n^{18}+2,158 n^{17}+6,983 n^{16}\right. \\
& +97,994 n^{15}+155,517 n^{14}-1,256,916 n^{13}-3,302,168 n^{12} \\
& +5,191,280 n^{11}+25,505,142 n^{10}+14,486,584 n^{9}-63,005,002 n^{8} \\
& -153,766,236 n^{7}-178,037,517 n^{6}-131,841,558 n^{5}-68,012,397 n^{4} \\
& \left.-24,910,146 n^{3}-6,269,211 n^{2}-975,888 n-70,470\right) \\
\geq & 0 .
\end{aligned}
$$

Thus, take $N=3$ and, for all $n \geq N$, we have $\delta(n) \geq 0, \delta^{2}(n) \geq \Delta(n)$, which follows from the inequality (3.1). We show the inequality (3.2) for some positive integer $M$. Note that, by Lemma 2.2 and some calculations, we have

$$
\begin{aligned}
f\left(g_{n}\right)= & c_{3}(n) g_{n}^{3}+c_{2}(n) g_{n}^{2}+c_{1}(n) g_{n}+c_{0}(n) \\
= & \frac{1,048,576}{n^{9}(n+1)^{6}(n+2)^{4}(n+3)^{2}}\left(54 n^{15}+378 n^{14}+916 n^{13}+644 n^{12}-1,529 n^{11}\right. \\
& -5,340 n^{10}-8,383 n^{9}-7,416 n^{8}-2,284 n^{7}+4,156 n^{6}+7,969 n^{5}+7,688 n^{4} \\
& \left.+4,953 n^{3}+2,154 n^{2}+576 n+72\right) .
\end{aligned}
$$

Take $M=6$, it is not difficult to verify that, for all $n \geq M$,

$$
f\left(g_{n}\right)>0 \text {. }
$$

Let $N_{0}=\max \{N, M\}=6$, then for all $n \geq 6$, all of the above inequalities hold. By Lemma 3.1 and Theorem 1.2, the Catalan-Larcombe-French sequence $\left\{P_{n}\right\}_{n \geq 6}$ is strictly 2-log-convex for all $n \geq 6$. What is more, one can easily test that these numbers $\left\{P_{n}\right\}_{0 \leq n \leq 8}$ also satisfy the property of 2-log-convexity by simple calculations. Therefore, the whole sequence $\left\{P_{n}\right\}_{n \geq 0}$ is strictly 2-log-convex. This completes the proof.

It deserves to be mentioned that by considerable calculations and plenty of verifications, the following conjectures should be true. 
Conjecture 3.2 The Catalan-Larcombe-French sequence is $\infty$-log-convex.

Conjecture 3.3 The quotient sequence $\left\{\frac{P_{n}}{P_{n-1}}\right\}_{n \geq 1}$ of the Catalan-Larcombe-French sequence is log-concave, equivalently, for all $n \geq 2$,

$$
P_{n-2} P_{n}^{3} \geq P_{n+1} P_{n-1}^{3} .
$$

\section{Competing interests}

The authors declare that they have no competing interests.

\section{Authors' contributions}

All authors contributed equally to the writing of this paper. All authors read and approved the final manuscript.

\section{Acknowledgements}

Research supported by NSFC (No. 11161046) and by the Xingjiang Talent Youth Project (No. 2013721012).

Received: 14 August 2015 Accepted: 30 November 2015 Published online: 18 December 2015

\section{References}

1. Brenti, F: Unimodal, log-concave, and Pólya frequency sequences in combinatorics. Mem. Am. Math. Soc. 413, 1-106 (1989)

2. Stanley, RP: Log-concave and unimodal sequences in algebra, combinatorics, and geometry. Ann. N.Y. Acad. Sci. 576, 500-535 (1989)

3. Jarvis, F, Verrill, HA: Supercongruences for the Catalan-Larcombe-French numbers. Ramanujan J. 22, 171-186 (2010). doi:10.1007/s11139-009-9218-5

4. Zhao, F-Z: The log-behavior of the Catalan-Larcombe-French sequences. Int. J. Number Theory 10, 177-182 (2014)

5. Sloane, NJA: The on-line encyclopedia of integer sequences. https://oeis.org/

6. Larcombe, PJ, French, DR: On the 'other' Catalan numbers: a historical formulation re-examined. Congr. Numer. 143 33-64 (2000)

7. Chen, WYC, Xia, EXW: The 2-log-convexity of the Apéry numbers. Proc. Am. Math. Soc. 139, 391-400 (2011)

8. Liu, LL, Wang, Y: On the log-convexity of combinatorial sequences. Adv. Appl. Math. 39, 453-476 (2007)

9. Chen, WYC, Guo, JJF, Wang, LXW: Infinitely log-monotonic combinatorial sequences. Adv. Appl. Math. 52, 99-120 (2014)

10. Chen, WYC, Guo, JJF, Wang, LXW: Zeta functions and the log-behavior of combinatorial sequences. Proc. Edinb. Math. Soc. (2). To appear. arXiv:1208.5213

11. Liggett, TM: Ultra logconcave sequences and negative dependence. J. Comb. Theory, Ser. A 79, 315-325 (1997)

12. Došlić, T: Log-balanced combinatorial sequences. Int. J. Math. Math. Sci. 4, 507-522 (2005)

\section{Submit your manuscript to a SpringerOpen ${ }^{\circ}$ journal and benefit from:}

- Convenient online submission

Rigorous peer review

- Immediate publication on acceptance

Open access: articles freely available online

- High visibility within the field

- Retaining the copyright to your article 